\title{
Intramolecular Cyclization of Ene-Imine Using Dibutylzirconocene
}

\author{
Muneyoshi Makabe, Yoshihiro Sato and Miwako Mori* \\ Graduate School of Pharmaceutical Sciences, Hokkaido University, \\ Sapporo 060-0812, Japan
}

Contents of Supporting Information

Experimental Section

S1-S17

General. All manipulations were performed under an argon atmosphere unless otherwise mentioned. Solvents were distilled under an argon atmosphere from sodium-benzophenone (THF, $\mathrm{Et}_{2} \mathrm{O}$, toluene, and benzene), $\mathrm{CaH}_{2}\left(\mathrm{CH}_{2} \mathrm{Cl}_{2}\right)$, or sodium (EtOH, MeOH). All other solvents and reagents were purified when necessary using standard procedures. Column chromatography was performed on silica gel 60 (Merck, 70-230 mesh), and flash chromatography was performed on silica gel 60 (Merck, 230-400 mesh) using the indicated solvent.

General Procedure for the Synthesis of Imines (1a-e) from Aldehydes (3a) and Amines. A solution of ene-aldehyde 3a (1 equiv) and amine (1 equiv) in benzene (0.1 M) was stirred in the presence of $\mathrm{MgSO}_{4}$ (10 equiv) at room temperature overnight. The solution was filtered, and the filtrate was concentrated. The residue was used 
without further purification.

$N$-(3,3-bis(benzyloxymethyl)hex-5-enylidene)(phenyl)methanamine $\quad(1 \mathrm{a}): \quad$ IR (neat) v 3063, 3028, 2857, 1662, 1495, 1453, 1364, $1099 \mathrm{~cm}^{-1} ;{ }^{1} \mathrm{H}$ NMR $(400 \mathrm{MHz}$, $\left.\mathrm{CDCl}_{3}\right) \delta 2.21(\mathrm{~d}, J=7.6 \mathrm{~Hz}, 2 \mathrm{H}), 2.39(\mathrm{~d}, J=5.6 \mathrm{~Hz}, 2 \mathrm{H}), 3.39(\mathrm{~d}, J=2.8 \mathrm{~Hz}, 4 \mathrm{H})$, 4.45 (s, 4 H), 4.49 (s, 2 H), 5.00-5.02 (m, 1 H), 5.04 (s, 1 H), 5.74-5.84 (m, 1 H), 7.83 (dt, $J=1.6,5.6 \mathrm{~Hz}, 1 \mathrm{H}) ;{ }^{13} \mathrm{C} \mathrm{NMR}\left(100 \mathrm{MHz}, \mathrm{CDCl}_{3}\right) \delta 37.7,39.9,42.4,65.3,73.0$, 73.2, 118.1, 126.7, 127.3 (x 2), 127.9 (x 4), 128.2 (x 4), 128.3 (x 2), 128.3 (x 2), 133.8 (x 2), 138.5, 164.3; LRMS (EI) $m / z, 428\left(\mathrm{M}^{+}+\mathrm{H}\right), 386,350,336,306,133,91$; HRMS (EI) calcd for $\mathrm{C}_{29} \mathrm{H}_{33} \mathrm{NO}_{2}\left(\mathrm{M}^{+}\right)$427.2511, found 427.2520.

$N$-(3,3-bis(benzyloxymethyl)hex-5-enylidene)methanamine (1b): IR (neat) $v$ 3062, 3029, 2857, 1667, $1099 \mathrm{~cm}^{-1}$; ${ }^{1} \mathrm{H}$ NMR (400 MHz, $\left.\mathrm{CDCl}_{3}\right) \delta 2.20(\mathrm{~d}, J=7.6 \mathrm{~Hz}, 2 \mathrm{H})$, $2.32(\mathrm{dd}, J=1.2,5.4 \mathrm{~Hz}, 2 \mathrm{H}), 3.02(\mathrm{bd}, J=1.2 \mathrm{~Hz}, 3 \mathrm{H}), 3.35(\mathrm{~d}, J=9.2 \mathrm{~Hz}, 2 \mathrm{H})$, $3.41(\mathrm{~d}, J=9.2 \mathrm{~Hz}, 2 \mathrm{H}), 4.48(\mathrm{~s}, 2 \mathrm{H}), 4.48(\mathrm{~s}, 2 \mathrm{H}), 5.02-5.06(\mathrm{~m}, 2 \mathrm{H}), 5.74-5.84$ (m, $1 \mathrm{H}), 7.23-7.34(\mathrm{~m}, 10 \mathrm{H}), 7.69(\mathrm{dt}, J=1.6,5.2 \mathrm{~Hz}, 1 \mathrm{H}) ;{ }^{13} \mathrm{C}$ NMR $(100 \mathrm{MHz}$, $\mathrm{CDCl}_{3}$ ) $\delta 37.6,39.7,42.3,48.0,73.0$ (x 2), 73.2 (x 2), 118.0, 127.3 (x 4), 128.2 (x 2), 133.8 (x 2), 138.6, 164.3; LRMS (EI) $m / z 352\left(\mathrm{M}^{+}+\mathrm{H}\right), 336,260,230,107,91,57$; HRMS (EI) calcd for $\mathrm{C}_{23} \mathrm{H}_{29} \mathrm{NO}_{2}\left(\mathrm{M}^{+}\right) 351.2198$, found 351.2196.

$N$-(3,3-bis(benzyloxymethyl)hex-5-enylidene)propan-1-amine (1c): IR (neat) v 3065, 3030, 2927, 2857, 1664, 1454, $1100 \mathrm{~cm}^{-1} ;{ }^{1} \mathrm{H}$ NMR $\left(400 \mathrm{MHz}, \mathrm{CDCl}_{3}\right) \delta$ $0.85(\mathrm{t}, J=7.2 \mathrm{~Hz}, 3 \mathrm{H}), 1.56(\mathrm{qt}, J=7.2,7.2 \mathrm{~Hz}, 2 \mathrm{H}), 2.20(\mathrm{~d}, J=7.2 \mathrm{~Hz}, 2 \mathrm{H})$, $2.32(\mathrm{~d}, J=5.6 \mathrm{~Hz}, 1 \mathrm{H}), 3.26(\mathrm{t}, J=7.2 \mathrm{~Hz}, 3 \mathrm{H}), 3.39(\mathrm{dd}, J=9.2,10.4 \mathrm{~Hz}, 4 \mathrm{H})$, 
$4.48(\mathrm{~s}, 4 \mathrm{H}), 5.03(\mathrm{~s}, 1 \mathrm{H}), 5.06(\mathrm{bd}, J=2.4 \mathrm{~Hz}, 1 \mathrm{H}), 5.75-5.85(\mathrm{~m}, 1 \mathrm{H})$, 7.25-7.36 (m, 10H), $7.68(\mathrm{bt}, J=5.6 \mathrm{~Hz}, 1 \mathrm{H}) ;{ }^{13} \mathrm{C} \mathrm{NMR}\left(100 \mathrm{MHz}, \mathrm{CDCl}_{3}\right) \delta$ 11.9, 23.9, 37.5, $39.5,42.2,63.3,72.9,73.1,73.1,73.2,118.0,127.3,127.4,128.2,128.3,133.8$, 138.5, 162.8; LRMS (EI) $m / z 380\left(\mathrm{M}^{+}+\mathrm{H}\right), 350,338,288,258,107,91,85,70,56$, 43; HRMS calcd for $\mathrm{C}_{25} \mathrm{H}_{33} \mathrm{NO}_{2}\left(\mathrm{M}^{+}\right)$379.2511, found 379.2504.

$N$-(3,3-bis(benzyloxymethyl)hex-5-enylidene)propan-2-amine (1d): IR (neat) v 3064, 3030, 2965, 2858, 1664, $1099 \mathrm{~cm}^{-1} ;{ }^{1} \mathrm{H}$ NMR (400 MHz, $\left.\mathrm{CDCl}_{3}\right) \delta 1.10$ (d, J $=6.4 \mathrm{~Hz}, 6 \mathrm{H}), 2.20(\mathrm{~d}, J=7.6 \mathrm{~Hz}, 2 \mathrm{H}), 2.30(\mathrm{~d}, J=5.6 \mathrm{~Hz}, 2 \mathrm{H}), 3.20(\mathrm{qq}, J=6.4$, $6.4 \mathrm{~Hz}, 1 \mathrm{H}), 4.42(\mathrm{dd}, J=9.2,10.4 \mathrm{~Hz}, 4 \mathrm{H}), 4.48(\mathrm{~s}, 4 \mathrm{H}), 5.02(\mathrm{~s}, 1 \mathrm{H}), 5.05$ (bd, $J$ $=2.8 \mathrm{~Hz}, 1 \mathrm{H}), 5.75-5.85(\mathrm{~m}, 1 \mathrm{H}), 7.24-7.36(\mathrm{~m}, 10 \mathrm{H}), 7.70(\mathrm{t}, J=5.6 \mathrm{~Hz}, 1 \mathrm{H}) ;{ }^{13} \mathrm{C}$ NMR (100 MHz, $\left.\mathrm{CDCl}_{3}\right) \delta 24.1,37.6,39.5,42.3,61.4,72.7,73.0,73.2$ (x 2), 118.0,127.3, 127.4, 128.2, 128.3, 133.8, 138.6, 160.2; LRMS (EI) $\mathrm{m} / z 380\left(\mathrm{M}^{+}+\mathrm{H}\right)$, $364,338,288,258,107,91,85,70,43$; HRMS (EI) calcd for $\mathrm{C}_{25} \mathrm{H}_{29} \mathrm{NO}_{2}\left(\mathrm{M}^{+}+\mathrm{H}\right)$ 380.2589 , found 380.2591 .

\section{$N$-(3-(benzyloxy)methyl)-3-(benzyloxymethyl)hex-5-enylidene)-2-methylpropan-}

2-amine (1e): IR (neat) v 3065, 3031, 2966, 2860, 1661, 1212, $1099 \mathrm{~cm}^{-1} ;{ }^{1} \mathrm{H}$ NMR $\left(400 \mathrm{MHz}, \mathrm{CDCl}_{3}\right) \delta 1.10(\mathrm{~s}, 9 \mathrm{H}), 2.19(\mathrm{~d}, J=7.6 \mathrm{~Hz}, 2 \mathrm{H}), 2.32(\mathrm{~d}, J=5.2 \mathrm{~Hz}, 2 \mathrm{H})$, $3.38(\mathrm{~s}, 4 \mathrm{H}), 4.47(\mathrm{~s}, 1 \mathrm{H}), 5.02(\mathrm{~s}, 1 \mathrm{H}), 5.05(\mathrm{bd}, J=3.2 \mathrm{~Hz}, 1 \mathrm{H}), 5.76-5.86(\mathrm{~m}, 1$ $\mathrm{H}), 7.25-7.36(\mathrm{~m}, 10 \mathrm{H}), 7.67(\mathrm{t}, J=5.6 \mathrm{~Hz}, 1 \mathrm{H}) ;{ }^{13} \mathrm{C} \mathrm{NMR}\left(100 \mathrm{MHz}, \mathrm{CDCl}_{3}\right)$ $\delta 29.6,37.6,40.2,42.3,56.8,73.1,73.1,73.2,73.2,117.9,127.2,127.3,128.1,128.2$, 133.9, 138.5, 157.2; LRMS (EI) m/z $394\left(\mathrm{M}^{+}+\mathrm{H}\right)$, 378, 352, 302, 272, 107, 91, 84, 57, 
41; HRMS (EI) calcd for $\mathrm{C}_{26} \mathrm{H}_{36} \mathrm{NO}_{2}\left(\mathrm{M}^{+}+\mathrm{H}\right)$ 394.2746, found 394.2756.

General Procedure for the zirconium-mediated cyclization of ene-imine. To a solution of $\mathrm{Cp}_{2} \mathrm{ZrCl}_{2}$ (1.3 equiv) in THF $(0.1 \mathrm{M})$ was added BuLi (1.6 M solution in hexane, 2.6 equiv) at $-78{ }^{\circ} \mathrm{C}$ and the solution was stirred at the same temperature for $1 \mathrm{~h}$. To this solution was added ene-imine 1 (1 equiv) at $-78{ }^{\circ} \mathrm{C}$ and the solution was stirred at room temperature for the appropriate hours. Saturated $\mathrm{NH}_{4} \mathrm{Cl}$ solution was added, and the solution was stirred at room temperature for $2 \mathrm{~h}$. The aqueous layer was made basic by $\mathrm{K}_{2} \mathrm{CO}_{3}$, and extracted with EtOAc. The organic layer was washed with brine, dried over $\mathrm{Na}_{2} \mathrm{SO}_{4}$, and concentrated. The residue was purified by flash column chromatography on silica gel to give the cyclized product.

$N$-benzyl-4,4-bis(benzyloxymethyl)-2-methylcyclopentanamine (trans-2a, cis2a): trans-2a: IR (neat) v 2919, 2856, $1095 \mathrm{~cm}^{-1}$; ${ }^{1} \mathrm{H}$ NMR (400 MHz, $\mathrm{CDCl}_{3}$ ) $\delta 0.97(\mathrm{~d}, J=6.4 \mathrm{~Hz}, 3 \mathrm{H}), 1.10(\mathrm{dd}, J=10.6,12.4 \mathrm{~Hz}, 1 \mathrm{H}), 1.27(\mathrm{dd}, J=9.6,13.2$ $\mathrm{Hz}, 1 \mathrm{H}), 1.40(\mathrm{bs}, 1 \mathrm{H}), 1.71-1.78(\mathrm{~m}, 1 \mathrm{H}), 1.81(\mathrm{dd}, J=7.2,13.2 \mathrm{~Hz}, 1 \mathrm{H}), 2.04(\mathrm{dd}$, $J=7.2,13.2 \mathrm{~Hz}, 1 \mathrm{H}), 2.58(\mathrm{dd}, J=9.2,9.6 \mathrm{~Hz}, 1 \mathrm{H}), 3.33(\mathrm{~s}, 2 \mathrm{H}), 3.38(\mathrm{dd}, J=8.8$, $12.2 \mathrm{~Hz}, 2 \mathrm{H}), 3.67(\mathrm{~d}, J=13.2 \mathrm{~Hz}, 1 \mathrm{H}), 3.83(\mathrm{~d}, J=12.8 \mathrm{~Hz}, 1 \mathrm{H}), 4.51(\mathrm{~s}, 4 \mathrm{H})$, 7.22-7.34 (m, $15 \mathrm{H}) ;{ }^{13} \mathrm{C} \mathrm{NMR}\left(100 \mathrm{MHz}, \mathrm{CDCl}_{3}\right) \delta$ 17.9, 39.7, 39.8, 44.5, 52.7, 64.9, 73.1, 73.2, 75.2, 75.4, 126.7, 127.2 (x 2), 127.3 (x 4), 127.9 (x 2), 128.2 (x 4), 128.3 (x 2), 138.6 (x 2), 140.8; LRMS (FAB) $m / z 430\left(\mathrm{M}^{+}+\mathrm{H}\right), 338$, 91; HRMS (FAB) calcd for $\mathrm{C}_{29} \mathrm{H}_{36} \mathrm{NO}_{2}\left(\mathrm{M}^{+}+\mathrm{H}\right)$ 430.2746, found 430.2726. cis-2a: IR (neat) $v 2922$, 2856, $1095 \mathrm{~cm}^{-1} ;{ }^{1} \mathrm{H}$ NMR (400 MHz, $\left.\mathrm{CDCl}_{3}\right) \delta 0.93(\mathrm{~d}, J=7.2 \mathrm{~Hz}, 3 \mathrm{H}), 1.39$ (dd, 
$J=7.6,13.6 \mathrm{~Hz}, 1 \mathrm{H}), 1.58(\mathrm{dd}, J=6.0,13.2 \mathrm{~Hz}, 1 \mathrm{H}), 1.64-1.71(\mathrm{~m}, 2 \mathrm{H}), 2.10-2.20$ (m, $1 \mathrm{H}), 3.04(\mathrm{q}, J=6.0 \mathrm{~Hz}, 1 \mathrm{H}), 3.33(\mathrm{~s}, 2 \mathrm{H}), 3.45(\mathrm{~s}, 2 \mathrm{H}), 3.63(\mathrm{~d}, J=13.2 \mathrm{~Hz}, 1$

$\mathrm{H}), 3.75(\mathrm{~d}, J=13.2 \mathrm{~Hz}, 1 \mathrm{H}), 4.51(\mathrm{~d}, J=2.0 \mathrm{~Hz}, 4 \mathrm{H}), 7.22-7.34(\mathrm{~m}, 15 \mathrm{H}) ;{ }^{13} \mathrm{C}$ NMR (100 MHz, $\left.\mathrm{CDCl}_{3}\right) \delta 14.6,36.5,37.4,38.6,45.7,52.2,61.1,73.1,73.2,75.2$, 75.7, 126.6, 127.2 (x 2), 127.3 (x 4), 127.4 (x 2), 128.0 (x 4), 128.2 (x 2), 138.8 (x 2), 141.0; LRMS (FAB) $m / z 430\left(\mathrm{M}^{+}+\mathrm{H}\right), 338,91$; HRMS (FAB) calcd for $\mathrm{C}_{29} \mathrm{H}_{36} \mathrm{NO}_{2}$ $\left(\mathrm{M}^{+}+\mathrm{H}\right)$ 430.2746, found 430.2765.

\section{4,4-bis(benzyloxymethyl)-N,2-dimethylcyclopentanamine (trans-2b, cis-2b):}

trans-2b: IR (neat) v 3566, 2924, 2849, 1654, 1560, 1451, 1095, $1026 \mathrm{~cm}^{-1} ;{ }^{1} \mathrm{H}$ NMR (400 MHz, $\left.\mathrm{CDCl}_{3}\right) \delta 1.05(\mathrm{~d}, J=6.4 \mathrm{~Hz}, 3 \mathrm{H}), 1.22(\mathrm{dd}, J=10.0,13.4 \mathrm{~Hz}, 1$ H), $1.51(\mathrm{dd}, J=8.0,13.6 \mathrm{~Hz}, 1 \mathrm{H}), 1.86-1.93(\mathrm{~m}, 1 \mathrm{H}) 1.99-2.08(\mathrm{~m}, 2 \mathrm{H}), 2.40(\mathrm{~s}, 3$ H), $2.71(\mathrm{dd}, J=8.0,15.6 \mathrm{~Hz}, 1 \mathrm{H}), 3.37(\mathrm{~d}, J=6.8 \mathrm{~Hz}, 2 \mathrm{H}), 3.39(\mathrm{~d}, J=8.8 \mathrm{~Hz}, 2$ $\mathrm{H}), 3.37(\mathrm{dd}, J=8.4,16.0 \mathrm{~Hz}, 2 \mathrm{H}), 4.47-4.50(\mathrm{~m}, 4 \mathrm{H}), 7.24-7.34(\mathrm{~m}, 10 \mathrm{H}) ;{ }^{13} \mathrm{C}$ NMR (100 MHz, $\left.\mathrm{CDCl}_{3}\right) \delta 14.7,35.7,37.4,38.3,45.0,46.4,58.2,73.2$ (x2) 75.3, 75.9, 127.3 (x 4), 127.4 (x 2), 128.2 (x 4), 138.6, 138.8 ; LRMS (EI) $m / z 354$ (M+H), 276, 262, 232, 91; HRMS (EI) calcd for $\mathrm{C}_{23} \mathrm{H}_{31} \mathrm{NO}_{2}\left(\mathrm{M}^{+}\right)$353.2355, found 353.2337. cis-2b: IR (neat) $v$ 2955, 2925, 2855, 1453, 1362, 1099, $1028 \mathrm{~cm}^{-1} ;{ }^{1} \mathrm{H}$ NMR (400 $\left.\mathrm{MHz}, \mathrm{CDCl}_{3}\right) \delta 1.05(\mathrm{~d}, J=6.4 \mathrm{~Hz}, 3 \mathrm{H}), 1.43(\mathrm{dd}, J=8.4,13.6 \mathrm{~Hz}, 1 \mathrm{H}), 1.68(\mathrm{dd}, J$ $=5.2,14.0 \mathrm{~Hz}, 1 \mathrm{H}), 1.74-1.82(\mathrm{~m}, 2 \mathrm{H}), 2.18-2.24(\mathrm{~m}, 1 \mathrm{H}), 2.38(\mathrm{~s}, 3 \mathrm{H}), 3.07(\mathrm{dd}, J$ $=5.6,11.2 \mathrm{~Hz}, 1 \mathrm{H}), 3.28-3.47(\mathrm{~m}, 4 \mathrm{H}), 4.47-4.50(\mathrm{~m}, 4 \mathrm{H}), 7.23-7.34(\mathrm{~m}, 10 \mathrm{H}) ;{ }^{13} \mathrm{C}$ NMR (100 MHz, $\left.\mathrm{CDCl}_{3}\right) \delta 17.9,22.4,39.5,40.4,44.6,47.4,62.7,73.1,73.2,75.3$, 
75.5, 127.2 (x 2), 127.3 (x 2), 127.4 (x 2), 128.2 (x 4), 138.6, 138.8; LRMS (FAB) $m / z 354\left(\mathrm{M}^{+}+\mathrm{H}\right), 276,262,232,91$; HRMS (EI) calcd for $\mathrm{C}_{23} \mathrm{H}_{31} \mathrm{NO}_{2}\left(\mathrm{M}^{+}\right)$353.2355, found 353.2351 .

\section{4,4-bis(benzyloxymethyl)-2-methyl- $N$-propylcyclopentanamine (trans-2c, cis-2c):}

trans-2c: IR (neat) v 2953, 2855, 1654, 1560, 1452, 1099, $1028 \mathrm{~cm}^{-1} ;{ }^{1} \mathrm{H}$ NMR (400 $\left.\mathrm{MHz}, \mathrm{CDCl}_{3}\right) \delta 0.89(\mathrm{t}, J=7.2 \mathrm{~Hz}, 3 \mathrm{H}), 0.98(\mathrm{~d}, J=6.4 \mathrm{~Hz}, 3 \mathrm{H}), 1.11(\mathrm{dd}, J=10.8$, $12.6 \mathrm{~Hz}, 1 \mathrm{H}), 1.23(\mathrm{dd}, J=9.6,13.2 \mathrm{~Hz}, 1 \mathrm{H}), 1.46(\mathrm{qt}, J=7.6,7.6 \mathrm{~Hz}, 2 \mathrm{H}), 1.68-$ $1.77(\mathrm{~m}, 1 \mathrm{H}), 1.81(\mathrm{dd}, J=7.6,12.6 \mathrm{~Hz}, 1 \mathrm{H}), 1.99(\mathrm{dd}, J=6.4,13.2 \mathrm{~Hz}, 1 \mathrm{H}), 2.04$ (bs, $1 \mathrm{H}), 2.46-2.61(\mathrm{~m}, 3 \mathrm{H}), 3.33(\mathrm{~s}, 2 \mathrm{H}), 3.36(\mathrm{dd}, J=8.4,12.8 \mathrm{~Hz}, 2 \mathrm{H}), 4.50(\mathrm{~d}, J$ $=4.0 \mathrm{~Hz}, 2 \mathrm{H}), 7.24-7.34(\mathrm{~m}, 10 \mathrm{H}) ;{ }^{13} \mathrm{C} \mathrm{NMR}\left(100 \mathrm{MHz}, \mathrm{CDCl}_{3}\right) \delta 11.9,18.0,23.4$, $39.6,39.8,39.8,44.5,50.4,65.2,73.1,73.2,75.3,75.5,127.2$ (x 2), 127.3 (x 2), 127.4 (x 2), 128.2 (x 4), 138.7, 138.8 ; LRMS (EI) $m / z 381\left(\mathrm{M}^{+}\right), 380,366,290,91$; HRMS (EI) calcd for $\mathrm{C}_{25} \mathrm{H}_{35} \mathrm{NO}_{2}\left(\mathrm{M}^{+}\right) 381.2668$, found 381.2672. cis-2c: IR (neat) $v$ 2953, 2855, 1654, 1560, 1452, 1099, $1028 \mathrm{~cm}^{-1} ;{ }^{1} \mathrm{H}$ NMR (400 MHz, $\left.\mathrm{CDCl}_{3}\right) \delta 0.89$ (t, $J=7.2 \mathrm{~Hz}, 3 \mathrm{H}), 0.98(\mathrm{~d}, J=6.4 \mathrm{~Hz}, 3 \mathrm{H}), 1.22-1.24(\mathrm{~m}, 1 \mathrm{H}), 1.39(\mathrm{dd}, J=7.6$, 13.6 Hz, $1 \mathrm{H}), 1.44-1.48(\mathrm{~m}, 2 \mathrm{H}), 1.68-1.77(\mathrm{~m}, 1 \mathrm{H}), 1.81(\mathrm{dd}, J=7.6,12.6 \mathrm{~Hz}, 1$ H), $1.99(\mathrm{dd}, J=6.4,13.2 \mathrm{~Hz}, 1 \mathrm{H}), 2.10-2.20(\mathrm{~m}, 1 \mathrm{H}), 2.46-2.61(\mathrm{~m}, 3 \mathrm{H}), 3.04(\mathrm{q}, J$ $=6.0 \mathrm{~Hz}, 1 \mathrm{H}), 3.33(\mathrm{~s}, 2 \mathrm{H}), 3.36(\mathrm{dd}, J=8.4,12.8 \mathrm{~Hz}, 2 \mathrm{H}), 4.50(\mathrm{~d}, J=4.0 \mathrm{~Hz}, 2$ $\mathrm{H})$, 7.24-7.34 (m, $10 \mathrm{H}) ;{ }^{13} \mathrm{C} \mathrm{NMR}\left(100 \mathrm{MHz}, \mathrm{CDCl}_{3}\right) \delta$ 14.5, 37.0, 37.1, 38.5, 44.5, 50.4, 58.5, 58.7, 65.2, 73.1, 73.2, 75.3, 75.5, 127.2 (x 2), 127.3 (x 2), 127.4 (x 2), 128.2 (x 4), 138.7, 138.8 ; LRMS (EI) $m / z 381\left(\mathrm{M}^{+}\right), 380,366,290,91$; HRMS (EI) 
calcd for $\mathrm{C}_{25} \mathrm{H}_{35} \mathrm{NO}_{2}\left(\mathrm{M}^{+}\right)$381.2668, found 381.2675.

4,4-bis(benzyloxymethyl)- $N$-isopropyl-2-methylcyclopentanamine (trans-2d, cis2d): trans-2d :IR (neat) v 2955, 2925, 2855, 1453, 1362, 1099, $1028 \mathrm{~cm}^{-1} ;{ }^{1} \mathrm{H}$ NMR $\left(400 \mathrm{MHz}, \mathrm{CDCl}_{3}\right) \delta 0.98(\mathrm{~d}, J=6.4 \mathrm{~Hz}, 3 \mathrm{H}), 1.01(\mathrm{~d}, J=6.4 \mathrm{~Hz}, 3 \mathrm{H}), 1.05(\mathrm{~d}, J=$ $6.4 \mathrm{~Hz}, 3 \mathrm{H}), 1.11(\mathrm{dd}, J=11.2,13.2 \mathrm{~Hz}, 1 \mathrm{H}), 1.21(\mathrm{dd}, J=9.6,13.2 \mathrm{~Hz}, 1 \mathrm{H}), 1.65-$ $1.73(\mathrm{~m}, 1 \mathrm{H}), 1.81(\mathrm{dd}, J=7.6,13.2 \mathrm{~Hz}, 1 \mathrm{H}), 2.01(\mathrm{dd}, J=7.2,13.2 \mathrm{~Hz}, 1 \mathrm{H}), 2.61$ $(\mathrm{dt}, J=7.6,9.6 \mathrm{~Hz}, 1 \mathrm{H}), 2.83(\mathrm{qd}, J=6.4 \mathrm{~Hz}, 1 \mathrm{H}), 3.33(\mathrm{~s}, 2 \mathrm{H}), 3.36(\mathrm{dd}, J=8.8$, 13.6 Hz, $1 \mathrm{H}), 4.50$ (s, $2 \mathrm{H}), 4.51$ (s, $2 \mathrm{H}), 7.23-7.34$ (m, $10 \mathrm{H}) ;{ }^{13} \mathrm{C} \mathrm{NMR}(100 \mathrm{MHz}$, $\left.\mathrm{CDCl}_{3}\right) \delta 17.9,22.4,23.9,39.5,39.9,40.4,44.6,47.4,62.7,73.1,73.2,75.3,75.5$, 127.2 (x 2), 127.3 (x 2), 127.4 (x 2), 128.2 (x 4), 138.6, 138.8; LRMS (EI) $\mathrm{m} / \mathrm{z}$ 381 $\left(\mathrm{M}^{+}\right)$, 380, 366, 290, 91; HRMS (EI) calcd for $\mathrm{C}_{25} \mathrm{H}_{35} \mathrm{NO}_{2}\left(\mathrm{M}^{+}\right)$381.2668, found 381.2675. cis-2d: IR (neat) v 3566, 2924, 2849, 1654, 1560, 1451, 1095, $1026 \mathrm{~cm}^{-1}$; ${ }^{1} \mathrm{H}$ NMR (400 MHz, $\left.\mathrm{CDCl}_{3}\right) \delta 0.89(\mathrm{~d}, J=7.2 \mathrm{~Hz}, 3 \mathrm{H}), 1.02(\mathrm{~d}, J=6.4 \mathrm{~Hz}, 3 \mathrm{H})$, $1.03(\mathrm{~d}, J=6.4 \mathrm{~Hz}, 3 \mathrm{H}), 1.38(\mathrm{dd}, J=5.6,13.6 \mathrm{~Hz}, 1 \mathrm{H}), 1.44(\mathrm{dd}, J=8.2,13.2 \mathrm{~Hz}$, $1 \mathrm{H}), 1.68(\mathrm{dd}, J=7.6,13.6 \mathrm{~Hz}, 1 \mathrm{H}), 1.76(\mathrm{dd}, J=6.4,13.2 \mathrm{~Hz}, 1 \mathrm{H}), 1.84(\mathrm{bs}, 1 \mathrm{H})$, $2.14(\mathrm{ddd}, J=6.0,7.2,13.2 \mathrm{~Hz}, 1 \mathrm{H}), 2.81(\mathrm{td}, J=6.4,12.4 \mathrm{~Hz}, 1 \mathrm{H}), 3.17(\operatorname{td}, J=$ 6.0, $8.2 \mathrm{~Hz}, 1 \mathrm{H}), 3.31(\mathrm{~s}, 2 \mathrm{H}), 3.39$ (s, $2 \mathrm{H}), 4.50(\mathrm{~d}, J=2.0 \mathrm{~Hz}, 2 \mathrm{H}), 4.51(\mathrm{~s}, 2 \mathrm{H})$,

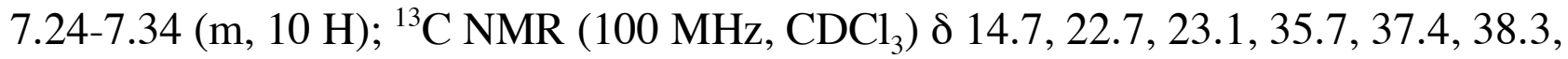
45.0, 46.4, 58.2, 73.2, 73.2, 75.3, 75.9, 127.3 (x 2), 127.3 (x 2), 127.4 (x 2), 128.2 (x 4), 138.6, 138.8; LRMS (EI) $m / z$ 381( $\left.{ }^{+}\right), 380,366,290$, 91; HRMS (EI) calcd for $\mathrm{C}_{25} \mathrm{H}_{35} \mathrm{NO}_{2}\left(\mathrm{M}^{+}\right)$381.2668, found 381.2672. 
4,4-bis(benzyloxymethyl)- $N$-tert-butyl-2-methylcyclopentanamine (cis-2 e): IR (neat) $\vee 2957,2860,1456,1387,1362,1229,1206,1097,1028 \mathrm{~cm}^{-1} ;{ }^{1} \mathrm{H}$ NMR $(400$ $\left.\mathrm{MHz}, \mathrm{CDCl}_{3}\right) \delta 0.87(\mathrm{~d}, J=7.2 \mathrm{~Hz}, 3 \mathrm{H}), 1.02(\mathrm{~s}, 9 \mathrm{H}), 1.33(\mathrm{dd}, J=5.6,13.6 \mathrm{~Hz}, 1$ H), $1.41(\mathrm{dd}, J=8.2,13.2 \mathrm{~Hz}, 1 \mathrm{H}), 1.48(\mathrm{bs}, 1 \mathrm{H}), 1.63(\mathrm{dd}, J=7.2,13.6 \mathrm{~Hz}, 1 \mathrm{H})$, $1.75(\mathrm{dd}, J=6.4,13.2 \mathrm{~Hz}, 1 \mathrm{H}), 1.96-2.02(\mathrm{~m}, 1 \mathrm{H}), 3.09(\mathrm{dd}, J=10.1,13.6 \mathrm{~Hz}, 1 \mathrm{H})$, 3.32 (s, $2 \mathrm{H}), 4.50$ (s, $2 \mathrm{H}), 4.51$ (s, $2 \mathrm{H}), 7.23-7.31$ (m, $10 \mathrm{H}) ;{ }^{13} \mathrm{C}$ NMR (100 MHz, $\left.\mathrm{CDCl}_{3}\right) \delta 15.1,30.1,37.6,38.4,40.9,44.8,50.6,73.1,73.2,75.5,76.0,127.2$ (x 2), 127.4 (x 4), 128.2 (x 4), 138.8 (x 2); LRMS (EI) m/z $395\left(\mathrm{M}^{+}\right)$, 380, 304, 91, 57; HRMS (EI) calcd for $\mathrm{C}_{26} \mathrm{H}_{37} \mathrm{NO}_{2}\left(\mathrm{M}^{+}\right)$395.2824, found 395.2811.

(2-(benzylamino)-4,4-bis(benzyloxymethyl)cyclopentyl)methanol (trans-6a, cis6a): To a solution of azazirconacycle 5a, which was prepared from $\mathrm{Cp}_{2} \mathrm{ZrCl}_{2}(95 \mathrm{mg}$, $0.325 \mathrm{mmol}$ ), BuLi (1.46 $\mathrm{M}$ in hexane, $0.44 \mathrm{~mL}, 0.65 \mathrm{mmol} \mathbf{1 a}(106.9 \mathrm{mg}, 0.25$ mmol), was added $\mathrm{NiCl}_{2}\left(\mathrm{PPh}_{3}\right)_{2}(327 \mathrm{mg}, 1.2 \mathrm{eq})$ at $0{ }^{\circ} \mathrm{C}$ and the whole mixture was stirred at room temperature for $6 \mathrm{~h}$. The argon atmosphere in the reaction vessel changed to oxygen and the mixture was stirred for $12 \mathrm{~h}$ under oxygen. Sat. $\mathrm{NH}_{4} \mathrm{Cl}$ solution was added at $0{ }^{\circ} \mathrm{C}$ and the mixture was stirred for $1 \mathrm{~h}$. The aqueous layer was made basic by $\mathrm{K}_{2} \mathrm{CO}_{3}$ and was extracted with ethyl acetate. The organic layer was washed with brine, dried over $\mathrm{Na}_{2} \mathrm{SO}_{4}$, and concentrated. The residue was purified by column chromatography on silica gel (hexane/ethyl acetate, 5/1-0/1) to give trans-6a (30.6 mg, 28\%) and cis-6a (13.1 mg, 12\%) as a colorless oil. trans-6a: IR (neat) $v 2919,2856,2360,2342,1095 \mathrm{~cm}^{-1} ;{ }^{1} \mathrm{H}$ NMR $\left(400 \mathrm{MHz}, \mathrm{CDCl}_{3}\right) \delta 1.08(\mathrm{dd}, J=$ 
10.6, 12.4 Hz, $1 \mathrm{H}), 1.19-1.28$ (m, $1 \mathrm{H}), 1.71-1.78$ (m, $2 \mathrm{H}), 2.01-2.06$ (m, $1 \mathrm{H}), 2.22$ (dd, $J=7.2,13.2 \mathrm{~Hz}, 1 \mathrm{H}), 2.43(\mathrm{bs}, 1 \mathrm{H}), 2.97$ (td, $J=10.0,7.2 \mathrm{~Hz}, 1 \mathrm{H}), 3.29$ (d, $J$ $=2.8 \mathrm{~Hz}, 2 \mathrm{H}), 3.36(\mathrm{~d}, J=2.8 \mathrm{~Hz}, 2 \mathrm{H}), 3.52(\mathrm{t}, J=10.0 \mathrm{~Hz}, 1 \mathrm{H}), 3.72(\mathrm{~d}, J=12.8$ $\mathrm{Hz}, 1 \mathrm{H}), 3.72(\mathrm{dd}, J=4.0,10.4 \mathrm{~Hz}, 1 \mathrm{H}), 3.86(\mathrm{~d}, J=12.8 \mathrm{~Hz}, 1 \mathrm{H}), 4.49$ (s, $2 \mathrm{H})$, 4.50 (s, $2 \mathrm{H})$, 7.22-7.34 (m, $15 \mathrm{H}) ;{ }^{13} \mathrm{C} \mathrm{NMR}\left(100 \mathrm{MHz}, \mathrm{CDCl}_{3}\right) \delta$ 29.8, 32.9, 39.6, $45.2,45.6,52.0,64.9,67.9,73.2,73.3,75.2,75.4,127.3$ (x 3), 127.4 (x 3), 128.1 (x 3), 128.2 (х 2), 128.3 (х 2), 128.5 (x 2), 138.4, 138.5, 138.7; LRMS (FAB) $m / z 446$ $\left(\mathrm{M}^{+}+1\right)$, 91; HRMS (FAB) calcd for $\mathrm{C}_{29} \mathrm{H}_{36} \mathrm{NO}_{3}\left(\mathrm{M}^{+}+\mathrm{H}\right) 446.2694$ found 446.2684. cis-6a: IR (neat) v 3340, 2921, 2854, 2360, 2342, $1097 \mathrm{~cm}^{-1} ;{ }^{1} \mathrm{H}$ NMR (400 MHz, $\left.\mathrm{CDCl}_{3}\right) \delta 1.08(\mathrm{dd}, J=10.6,12.4 \mathrm{~Hz}, 1 \mathrm{H}), 1.27(\mathrm{dd}, J=9.6,13.2 \mathrm{~Hz}, 1 \mathrm{H}), 1.71-$ $1.78(\mathrm{~m}, 2 \mathrm{H}), 1.81(\mathrm{dd}, J=7.2,13.2 \mathrm{~Hz}, 1 \mathrm{H}), 1.90-1.97(\mathrm{~m}, 2 \mathrm{H}), 2.04(\mathrm{dd}, J=7.2$, $13.2 \mathrm{~Hz}, 1 \mathrm{H}), 2.58(\mathrm{dd}, J=9.2,9.6 \mathrm{~Hz}, 1 \mathrm{H}), 3.33(\mathrm{~s}, 2 \mathrm{H}), 3.38(\mathrm{~d}, J=3.6 \mathrm{~Hz}, 2 \mathrm{H})$, 3.42-3.50 (m, $2 \mathrm{H}), 3.67(\mathrm{~d}, J=13.2 \mathrm{~Hz}, 1 \mathrm{H}), 3.83(\mathrm{~d}, J=12.8 \mathrm{~Hz}, 1 \mathrm{H}), 4.50(\mathrm{~s}, 2$ $\mathrm{H}), 4.51(\mathrm{~s}, 2 \mathrm{H}), 7.22-7.34(\mathrm{~m}, 15 \mathrm{H}) ;{ }^{13} \mathrm{C} \mathrm{NMR}\left(100 \mathrm{MHz}, \mathrm{CDCl}_{3}\right) \delta$ 29.7, 32.9, $38.0,45.1,45.3,51.2,63.8,66.5,73.2,73.3,75.1,75.2,127.3$ (x 3), 127.4 (x 2), 127.5 (x 2), 128.0 (x 2), 128.3 (x 2), 128.6 (x 2), 128.9 (x 2), 138.2, 138.3 (x 2); LRMS (FAB) $m / z 446\left(\mathrm{M}^{+}+\mathrm{H}\right), 354,154,107,91$; HRMS (FAB) calcd for $\mathrm{C}_{29} \mathrm{H}_{36} \mathrm{NO}_{3}$ $\left(\mathrm{M}^{+}+\mathrm{H}\right) 446.2694$ found 446.2694 .

4,4-bis(benzyloxymethyl)-2-(but-3-enyl)- $N$-tert-butylcyclopentanamine (7e): To a solution of azazirconacycle 5e, which was prepared from $\mathrm{Cp}_{2} \mathrm{ZrCl}_{2}(95 \mathrm{mg}, 0.325$ mmol) in THF $(0.9 \mathrm{~mL})$, BuLi $(1.46 \mathrm{M}$ in hexane, $0.44 \mathrm{~mL}, 0.65 \mathrm{mmol})$ and $1 \mathrm{e}$ 
(106.9 mg, $0.25 \mathrm{mmol})$ in THF $(1.0 \mathrm{~mL})$ was added $\mathrm{CuCl}(49.7 \mathrm{mg}, 0.513 \mathrm{mmol})$ and allyl chloride $(41 \mu \mathrm{l}, 0.513 \mathrm{mmol})$ in THF $(0.5 \mathrm{~mL})$ at $0{ }^{\circ} \mathrm{C}$, and the solution was stirred at room temperature for $1 \mathrm{~h}$. After saturated $\mathrm{NH}_{4} \mathrm{Cl}$ solution was added at $0{ }^{\circ} \mathrm{C}$, the mixture was stirred for $1 \mathrm{~h}$. The aqueous layer was made basic by $\mathrm{K}_{2} \mathrm{CO}_{3}$. The aqueous layer was extracted with EtOAc, and the organic layer was washed with brine, dried over $\mathrm{Na}_{2} \mathrm{SO}_{4}$, and concentrated. The residue was purified by column chromatography on silica gel (hexane/EtOAc $=4 / 1-2 / 1)$ to give $7 \mathbf{e}(61.8 \mathrm{mg}, 57 \%)$ as a pale yellow oil. 7e: IR (neat) v 2957, 2860, 1456, 1387, 1362, 1229, 1206, 1097, $1028 \mathrm{~cm}^{-1} ;{ }^{1} \mathrm{H}$ NMR $\left(400 \mathrm{MHz}, \mathrm{CDCl}_{3}\right) \delta 0.98(\mathrm{~s}, 9 \mathrm{H}), 1.22-1.31(\mathrm{~m}, 3 \mathrm{H}), 1.39(\mathrm{dd}$, $J=9.2,13.2 \mathrm{~Hz}, 1 \mathrm{H}), 1.47(\mathrm{dd}, J=5.0,13.6 \mathrm{~Hz}, 1 \mathrm{H}), 1.56(\mathrm{dd}, J=7.0,13.6 \mathrm{~Hz}, 1$ H), $1.69(\mathrm{dd}, J=6.0,13.2 \mathrm{~Hz}, 1 \mathrm{H}), 1.73-1.79(\mathrm{~m}, 1 \mathrm{H}), 1.94-2.08(\mathrm{~m}, 2 \mathrm{H}), 3.06(\mathrm{dd}$, $J=6.0,11.0 \mathrm{~Hz}, 1 \mathrm{H}), 3.29(\mathrm{~d}, J=2.8 \mathrm{~Hz}, 2 \mathrm{H}), 3.42(\mathrm{~d}, J=2.8 \mathrm{~Hz}, 2 \mathrm{H}), 4.50(\mathrm{~s}, 2$ H), $4.51(\mathrm{~s}, 2 \mathrm{H}), 4.90$ (tdd, $J=1.2,2.0,10.0 \mathrm{~Hz}, 1 \mathrm{H}), 4.97$ (ddd, $J=1.6,3.6,16.8$ $\mathrm{Hz}, 1 \mathrm{H}), 5.79(\mathrm{tdd}, J=6.8,10.0,16.8 \mathrm{~Hz}, 1 \mathrm{H}), 7.24-7.35(\mathrm{~m}, 10 \mathrm{H}) ;{ }^{13} \mathrm{C} \mathrm{NMR}(100$ $\left.\mathrm{MHz}, \mathrm{CDCl}_{3}\right) \delta 28.3,30.2,32.6,35.7,41.8,43.3,45.3,50.4,54.8,73.1,73.3,75.7$, 75.9, 113.9, 127.2, 127.3, 127.3, 127.6, 128.2, 138.7, 138.8, 139.4; LRMS (EI) m/z $435\left(\mathrm{M}^{+}\right), 420,394,378,344,91,57$; HRMS (EI) calcd for $\mathrm{C}_{29} \mathrm{H}_{41} \mathrm{NO}_{2}\left(\mathrm{M}^{+}\right)$435.3165, found 435.3151 .

\section{Zirconium-Mediated Diastereoselective Cyclization.}

(R)- $N$-(3,3-bis(benzyloxymethyl)hex-5-enylidene)-1-phenylethanamine $\quad(1 \mathrm{f}): \quad$ IR (neat) $v 3028,2969,2858,1661,1495,1452,1364,1099,1028 \mathrm{~cm}^{-1} ;{ }^{1} \mathrm{H}$ NMR (400 
$\left.\mathrm{MHz}, \mathrm{CDCl}_{3}\right) \delta 1.44(\mathrm{~d}, J=6.8 \mathrm{~Hz}, 3 \mathrm{H}), 2.18(\mathrm{~d}, J=7.2 \mathrm{~Hz}, 2 \mathrm{H}), 2.36(\mathrm{~d}, J=5.6$ $\mathrm{Hz}, 3 \mathrm{H}), 3.35(\mathrm{dd}, J=9.2,15.6 \mathrm{~Hz}, 2 \mathrm{H}), 3.36(\mathrm{~s}, 2 \mathrm{H}), 4.21(\mathrm{q}, J=6.8 \mathrm{~Hz}, 1 \mathrm{H})$, $4.42(\mathrm{dd}, J=12.0,14.0 \mathrm{~Hz}, 2 \mathrm{H}), 4.44(\mathrm{~s}, 2 \mathrm{H}), 5.78(\mathrm{tdd}, J=7.2,11.2,16.0 \mathrm{~Hz}, 1 \mathrm{H})$, $4.99(\mathrm{dd}, J=2.4,7.2 \mathrm{~Hz}, 1 \mathrm{H}), 5.03(\mathrm{~s}, 1 \mathrm{H}), 7.25-7.36(\mathrm{~m}, 15 \mathrm{H}), 7.80(\mathrm{t}, J=5.6 \mathrm{~Hz}$, $1 \mathrm{H}) ;{ }^{13} \mathrm{C} \mathrm{NMR}\left(100 \mathrm{MHz}, \mathrm{CDCl}_{3}\right) \delta$ 24.4, 37.6, 39.5, 42.0, 69.8, 72.9, 73.0, 73.2, 73.2, 118.0, 126.5 (x 3), 126.6 (x 3), 127.3 (x 3), 128.1 (x 3), 128.2 (x 3), 133.8, 138.6, 144.9, 161.9; LRMS (EI) $m / z$ 441( $\left.\mathrm{M}^{+}\right), 440,426,400,350,91$; HRMS (EI) calcd for $\mathrm{C}_{30} \mathrm{H}_{35} \mathrm{NO}_{2}\left(\mathrm{M}^{+}+\mathrm{H}\right)$ 442.2746, found 442.2727.

cis-4,4-bis(benzyloxymethyl)-2-methyl- $N$-((R)-1-phenylethyl)cyclopentanamine (8f): IR (neat) $v$ 3027, 2924, 2856, 1496, 1452, 1362, 1201, 1095, $1028 \mathrm{~cm}^{-1} ;{ }^{1} \mathrm{H}$ NMR (400 MHz, $\left.\mathrm{CDCl}_{3}\right) \delta 0.92(\mathrm{~d}, J=6.8 \mathrm{~Hz}, 3 \mathrm{H}), 1.19(\mathrm{~d}, J=6.8 \mathrm{~Hz}, 3 \mathrm{H}), 1.37$ $(\mathrm{dd}, J=8.8,13.2 \mathrm{~Hz}, 1 \mathrm{H}), 1.48(\mathrm{bs}, 1 \mathrm{H}), 1.56(\mathrm{dd}, J=6.0,13.6 \mathrm{~Hz}, 1 \mathrm{H}), 1.60-1.64$ (m, $2 \mathrm{H}), 1.93(\mathrm{qd}, J=7.2,7.2,1 \mathrm{H}), 2.74(\mathrm{dd}, J=5.6,10.8 \mathrm{~Hz}, 1 \mathrm{H}), 3.25(\mathrm{~s}, 2 \mathrm{H})$, $3.44(\mathrm{~s}, 2 \mathrm{H}), 3.73(\mathrm{dd}, J=6.8,13.2 \mathrm{~Hz}, 1 \mathrm{H}), 4.46(\mathrm{dd}, J=12.8,14.0 \mathrm{~Hz}, 2 \mathrm{H}), 4.53$ (s, $2 \mathrm{H})$, 7.18-7.34 (m, $15 \mathrm{H}) ;{ }^{13} \mathrm{C}$ NMR (100 MHz, $\left.\mathrm{CDCl}_{3}\right) \delta$ 14.5, 24.9, 36.9, 37.4, $38.4,45.8,55.5,58.7,73.1,73.3,75.5,75.9,126.5$ (x 2), 126.7 (x 2), 127.2 (x 3), 127.3 (x 2), 127.5 (x 2), 128.1 (x 2), 128.2 (x 2), 138.7, 138.8, 146.3; LRMS (EI) $\mathrm{m} / \mathrm{z}$ $442\left(\mathrm{M}^{+}-\mathrm{H}\right), 428,352,105,91$; HRMS (EI) calcd for $\mathrm{C}_{30} \mathrm{H}_{37} \mathrm{NO}_{2}\left(\mathrm{M}^{+}\right)$443.2829, found 443.2826 .

trans-4,4-bis(benzyloxymethyl)-2-methyl- $N-((R)-1-$

phenylethyl)cyclopentanamine (8f'): IR (neat) $v$ 3027, 2924, 2856, 1496, 1452, 
1362, 1201, 1095, $1028 \mathrm{~cm}^{-1}$; ${ }^{1} \mathrm{H}$ NMR (400 MHz, $\left.\mathrm{CDCl}_{3}\right) \delta 0.99(\mathrm{~d}, J=6.4 \mathrm{~Hz}, 3 \mathrm{H})$, $1.02-1.11(\mathrm{~m}, 2 \mathrm{H}), 1.30(\mathrm{~d}, J=6.8 \mathrm{~Hz}, 3 \mathrm{H}), 1.60-1.71(\mathrm{~m}, 1 \mathrm{H}), 1.75-1.81(\mathrm{~m}, 2 \mathrm{H})$, $2.55(\mathrm{dd}, J=9.2,16.8,1 \mathrm{H}), 3.25(\mathrm{~s}, 2 \mathrm{H}), 3.30(\mathrm{dd}, J=8.8,13.2 \mathrm{~Hz}, 2 \mathrm{H}), 3.83(\mathrm{dd}$, $J=6.8,12.8 \mathrm{~Hz}, 1 \mathrm{H}), 4.46(\mathrm{~d}, J=3.6 \mathrm{~Hz}, 4 \mathrm{H}), 7.19-7.32(\mathrm{~m}, 15 \mathrm{H}) ;{ }^{13} \mathrm{C}$ NMR $(100$ $\left.\mathrm{MHz}, \mathrm{CDCl}_{3}\right) \delta 18.2,23.9,39.6,40.6,40.8,44.4,57.1,63.6,73.1$ (x 2), 75.3, 75.5, 126.5 (x 3), 126.7 (x 4), 127.2 (x 4), 128.2 (x 4), 138.8, 138.9, 146.7; LRMS (EI) $\mathrm{m} / \mathrm{z}$ $442\left(\mathrm{M}^{+}-\mathrm{H}\right), 428,352,105,91$; HRMS (EI) calcd for $\mathrm{C}_{25} \mathrm{H}_{35} \mathrm{NO}_{2}\left(\mathrm{M}^{+}\right)$443.2829, found 443.2827 .

trans-4,4-bis(benzyloxymethyl)-2-methyl- $N-((R)-1-$

phenylethyl)cyclopentanamine (8f): IR (neat) v 3028, 2923, 2855, 1496, 1452, 1362, 1201, 1099, $1028 \mathrm{~cm}^{-1}$; ${ }^{1} \mathrm{H}$ NMR (400 MHz, $\left.\mathrm{CDCl}_{3}\right) \delta 0.90(\mathrm{~d}, J=6.4 \mathrm{~Hz}, 3 \mathrm{H})$, $0.95(\mathrm{t}, J=12.0 \mathrm{~Hz}, 1 \mathrm{H}), 1.19(\mathrm{dd}, J=9.8,12.8 \mathrm{~Hz}, 3 \mathrm{H}), 1.37(\mathrm{dd}, J=8.8,13.2 \mathrm{~Hz}$, $1 \mathrm{H}), 1.48$ (bs, $1 \mathrm{H}), 1.56(\mathrm{dd}, J=6.0,13.6 \mathrm{~Hz}, 1 \mathrm{H}), 1.60-1.64(\mathrm{~m}, 2 \mathrm{H}), 1.93$ (qd, $J$ = 7.2, 7.2, $1 \mathrm{H}), 2.74(\mathrm{dd}, J=5.6,10.8 \mathrm{~Hz}, 1 \mathrm{H}), 3.25(\mathrm{~s}, 2 \mathrm{H}), 3.44(\mathrm{~s}, 2 \mathrm{H}), 3.73(\mathrm{dd}$, $J=6.8,13.2 \mathrm{~Hz}, 1 \mathrm{H}), 4.46(\mathrm{dd}, J=12.8,14.0 \mathrm{~Hz}, 2 \mathrm{H}), 4.53(\mathrm{~s}, 2 \mathrm{H}), 7.18-7.34(\mathrm{~m}$, $15 \mathrm{H}) ;{ }^{13} \mathrm{C}$ NMR $\left(100 \mathrm{MHz}, \mathrm{CDCl}_{3}\right) \delta 17.5,25.5,39.5,39.9,40.0,44.5,56.4,62.3$, 73.1, 73.2, 75.2, 75.4, 126.3, 126.6 (x 2), 127.2 (x 2), 127.3 (x 2), 127.4 (x 2), 128.1, 128.2 (х 2), 138.7, 138.8, 145.7; LRMS (EI) $m / z 442\left(\mathrm{M}^{+}-\mathrm{H}\right), 428,352,105,91$; HRMS (EI) calcd for $\mathrm{C}_{25} \mathrm{H}_{35} \mathrm{NO}_{2}\left(\mathrm{M}^{+}\right)$443.2829, found 443.2827.

cis-4,4-bis(benzyloxymethyl)-2-methyl- $N$-((R)-1-phenylethyl)cyclopentanamine (8f'): IR (neat) v 3027, 2924, 2858, 1458, 1362, $1095 \mathrm{~cm}^{-1} ;{ }^{1} \mathrm{H}$ NMR (400 MHz, 
$\left.\mathrm{CDCl}_{3}\right) \delta 0.91(\mathrm{~d}, J=7.2 \mathrm{~Hz}, 3 \mathrm{H}), 1.29(\mathrm{~d}, J=6.4 \mathrm{~Hz}, 3 \mathrm{H}), 1.32-1.40(\mathrm{~m}, 2 \mathrm{H}), 1.59$ $(\mathrm{dd}, J=7.4,13.6 \mathrm{~Hz}, 1 \mathrm{H}), 1.66(\mathrm{dd}, J=6.4,13.2 \mathrm{~Hz}, 1 \mathrm{H}), 2.14(\mathrm{qd}, J=5.8,5.8,1$ H), $2.97(\mathrm{td}, J=6.0,8.4 \mathrm{~Hz}, 1 \mathrm{H}), 3.22(\mathrm{dd}, J=8.4,11.0,2 \mathrm{H}), 3.36(\mathrm{~s}, 2 \mathrm{H}), 3.75(\mathrm{q}$, $J=6.8 \mathrm{~Hz}, 1 \mathrm{H}), 4.43(\mathrm{dd}, J=12.4,14.4 \mathrm{~Hz}, 2 \mathrm{H}), 4.46(\mathrm{~s}, 2 \mathrm{H}), 7.20-7.34(\mathrm{~m}, 15 \mathrm{H})$; ${ }^{13} \mathrm{C}$ NMR $\left(100 \mathrm{MHz}, \mathrm{CDCl}_{3}\right) \delta 14.8,24.5,35.1,37.9,38.4,44.7,56.2,58.5,73.0$, 73.1, 75.1, 75.9, 126.6 (x 3), 127.2 (x 4), 127.3 (x 2), 128.1 (x 2), 128.2 (x 4), 138.8 (x 2), 146.1; LRMS (EI) $m / z, 442\left(\mathrm{M}^{+}-\mathrm{H}\right), 428,352,105,91$; HRMS (EI) calcd for $\mathrm{C}_{25} \mathrm{H}_{35} \mathrm{NO}_{2}\left(\mathrm{M}^{+}+\mathrm{H}\right)$ 444.2903, found 444.2891.

Conversion of 11 into tras-12 and tras-12', cis-12 and cis-12'. A solution of 11 $(72.8 \mathrm{mg}, 0.25 \mathrm{mmol})$ and $\mathrm{Pd}(\mathrm{OH})_{2}$ on charcoal $(20 \%, 17.5 \mathrm{mg}, 0.025 \mathrm{mmol})$ in $\mathrm{EtOH}(2.5 \mathrm{~mL})$ was stirred at room temperature for $9 \mathrm{~h}$ under an atmosphere of hydrogen. After the catalyst was filtered off, the solvent was evaporated to give the crude product, which was dissolved in $\mathrm{CH}_{2} \mathrm{Cl}_{2}(1 \mathrm{~mL})$. To this solution were added pyridine $(0.69 \mathrm{~mL}, 5.0 \mathrm{mmol})$ and $\mathrm{BzCl}(0.29 \mathrm{~mL}, 2.5 \mathrm{mmol})$ and the solution was stirred at room temperature for $12 \mathrm{~h}$. Ethyl acetate was added and the organic layer was washed saturated $\mathrm{NaHCO}_{3}$ and brine and dried over $\mathrm{Na}_{2} \mathrm{SO}_{4}$. After removal of the solvent, the residue was purified by column chromatography on silica gel (hexane/EtOAc, 2/1-1/2) to give trans-12 (12.2 mg, 18\% from 2) and cis-12 (54.8 mg, 75\% from 2). cis-12: IR (neat) $v$ 3362, 2955, 2926, 2854, 1663, 1540, $1107 \mathrm{~cm}^{-1} ;{ }^{1} \mathrm{H}$ NMR $\left(400 \mathrm{MHz}, \mathrm{CDCl}_{3}\right) \delta 0.97(\mathrm{~d}, J=6.8 \mathrm{~Hz}, 3 \mathrm{H}), 1.46-1.67(\mathrm{~m}, 3 \mathrm{H}), 1.88(\mathrm{dd}, J$ $=6.0,14.4 \mathrm{~Hz}, 1 \mathrm{H}), 2.08-2.19(\mathrm{~m}, 1 \mathrm{H}), 3.18(\mathrm{dd}, J=8.8,15.2 \mathrm{~Hz}, 2 \mathrm{H}), 3.33(\mathrm{~s}, 3$ 
H), $3.36(\mathrm{dd}, J=8.4,18.0 \mathrm{~Hz}, 2 \mathrm{H}), 3.47$ (s, $3 \mathrm{H}), 3.45-3.50(\mathrm{~m}, 1 \mathrm{H}), 3.40-3.49$ (m, 3 H), $7.75(\mathrm{bd}, J=8.0 \mathrm{~Hz}, 1 \mathrm{H}), 7.79-7.81(\mathrm{~m}, 2 \mathrm{H}) ;{ }^{13} \mathrm{C} \mathrm{NMR}\left(100 \mathrm{MHz}, \mathrm{CDCl}_{3}\right)$

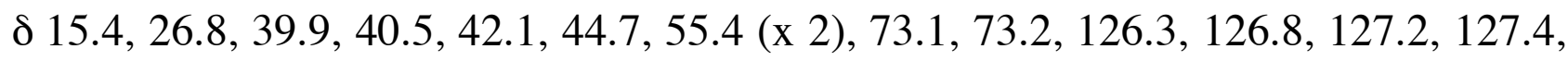
128.1, 138.7, 167.7; LRMS (EI) m/z $291\left(\mathrm{M}^{+}\right), 276,200,186,105,91$; HRMS (EI) calcd for $\mathrm{C}_{17} \mathrm{H}_{25} \mathrm{NO}_{3}\left(\mathrm{M}^{+}\right)$291.1834, found 291.1831. trans-12: IR (neat) $v 3028$, 2923, 2855, 1496, 1452, 1362, 1201, 1099, $1028 \mathrm{~cm}^{-1} ;{ }^{1} \mathrm{H}$ NMR (400 MHz, $\left.\mathrm{CDCl}_{3}\right) \delta$ $1.06(\mathrm{~d}, J=6.4 \mathrm{~Hz}, 3 \mathrm{H}), 1.22(\mathrm{dd}, J=6.8,12.8 \mathrm{~Hz}, 1 \mathrm{H}), 1.19(\mathrm{dd}, J=6.8,12.8 \mathrm{~Hz}$, $1 \mathrm{H}), 1.47(\mathrm{dd}, J=8.8,13.2 \mathrm{~Hz}, 1 \mathrm{H}), 1.93(\mathrm{dd}, J=7.6,13.2 \mathrm{~Hz}, 1 \mathrm{H}), 2.00(\mathrm{td}, J=$ 6.8, 14.0, $1 \mathrm{H}), 2.11(\mathrm{dd}, J=7.2,13.6 \mathrm{~Hz}, 1 \mathrm{H}), 3.24(\mathrm{dd}, J=8.8,14.4 \mathrm{~Hz}, 2 \mathrm{H}), 3.34$ (s, $3 \mathrm{H}), 3.40(\mathrm{~s}, 3 \mathrm{H}), 4.12(\mathrm{dd}, J=7.2,14.8 \mathrm{~Hz}, 1 \mathrm{H}), 6.80(\mathrm{bd}, J=8.0 \mathrm{~Hz}, 1 \mathrm{H})$, 7.40-7.52 (m, $3 \mathrm{H}), 7.76$ (bd, $2 \mathrm{H}) ;{ }^{13} \mathrm{C} \mathrm{NMR}\left(100 \mathrm{MHz}, \mathrm{CDCl}_{3}\right) \delta$ 17.5, 25.5, 39.5, $39.9,40.0,44.5,56.4$ (х 2), 73.1, 73.2, 126.6, 127.2, 127.3, 127.4, 128.1, 138.8, 165.7; LRMS (EI) m/z LRMS (EI) $m / z 291\left(\mathrm{M}^{+}\right), 276,200,186,105,91$; HRMS (EI) calcd for $\mathrm{C}_{17} \mathrm{H}_{25} \mathrm{NO}_{3}\left(\mathrm{M}^{+}\right)$291.1834, found 291.1831.

The enantiomeric excesses of $\boldsymbol{c i s} \mathbf{- 1 2}$ and trans-12 were determined by HPLC analysis [trans-12; DAICEL CHIRALPAK AD (hexane/2-propanol 95/5), cis-12; DAICEL CHIRALPAK AS (hexane/2-propanol 9/1)].

Conversion of cis-11 into (S)-MTPA Ester cis-14 (cis-(S)-14): A solution of cis11 (14.6 mg, $0.035 \mathrm{mmol})$ and $\mathrm{Pd}(\mathrm{OH})_{2}$ on charcoal $(20 \%, 2.8 \mathrm{mg}, 0.004 \mathrm{mmol})$ in EtOH $(1.5 \mathrm{~mL})$ was stirred at room temperature for $2.5 \mathrm{~h}$ under an atmosphere of hydrogen. After the catalyst was filtered off, the solvent was evaporated to give the 
crude, which was dissolved in $\mathrm{CH}_{2} \mathrm{Cl}_{2}$. To this solution were added $(S)-(-)-\alpha-$ methoxy- $\alpha$-(trifluoromethyl)phenylacetyl chloride $(27 \mu \mathrm{g}, 0.14 \mathrm{mmol})$, and pyridine (41 $\mu \mathrm{g}, 0.58 \mathrm{mmol}$ ) at $0{ }^{\circ} \mathrm{C}$, and the mixture was stirred at room temperature for $1 \mathrm{~h}$. To the mixture was added $N, N^{\prime}$-dimethyl-1,3-propanediamine (18 $\left.\mu 1,0.14 \mathrm{mmol}\right)$ at $0{ }^{\circ} \mathrm{C}$ and the mixture was stirred at room temperature for $1 \mathrm{~h}$. After removal of the solvent, the residue was purified by preparative TLC on silica gel (hexane/Et $\mathrm{E}_{2} \mathrm{O} 5 / 1$ ) to give cis-(S)-14 (6.9 mg, 2 step 49\%). cis-(S)-14: IR (neat) v 3314, 2957, 2928, 2870, 1693, $1106 \mathrm{~cm}^{-1} ;{ }^{1} \mathrm{H}$ NMR (400 MHz, $\left.\mathrm{CDCl}_{3}\right) \delta 0.98(\mathrm{~d}, J=6.8 \mathrm{~Hz}, 3 \mathrm{H}), 1.41$ $(\mathrm{d}, J=17.2 \mathrm{~Hz}, 1 \mathrm{H}), 1.44(\mathrm{~d}, J=13.6 \mathrm{~Hz}, 1 \mathrm{H}), 1.56(\mathrm{dd}, J=8.0,13.6 \mathrm{~Hz}, 1 \mathrm{H})$, $1.77(\mathrm{dd}, J=6.0,14.0 \mathrm{~Hz}, 1 \mathrm{H}), 2.06-2.13(\mathrm{~m}, 1 \mathrm{H}), 3 . \mathrm{s} 12(\mathrm{dd}, J=8.8,18.0 \mathrm{~Hz}, 2 \mathrm{H})$, $3.21(\mathrm{dd}, J=8.8,9.6 \mathrm{~Hz}, 2 \mathrm{H}), 3.30(\mathrm{dd}, J=0.4,1.6 \mathrm{~Hz}, 6 \mathrm{H}), 3.52(\mathrm{bd}, J=1.2 \mathrm{~Hz}, 3$ H), 4.26-4.32 (m, $1 \mathrm{H}), 7.35-7.37(\mathrm{~m}, 3 \mathrm{H}), 7.59-7.61(\mathrm{~m}, 2 \mathrm{H}), 8.06(\mathrm{bd}, J=8.8 \mathrm{~Hz}$, $1 \mathrm{H}) ;{ }^{13} \mathrm{C} \mathrm{NMR}\left(100 \mathrm{MHz}, \mathrm{CDCl}_{3}\right) \delta 14.2,37.7,39.7,40.1,46.5,53.8,54.8,58.9$, 59.3, 77.9, 79.3, 116.0, 124.2, 127.5, 128.0, 129.0, 133.6, 165.3 ; LRMS (EI) $\mathrm{m} / \mathrm{z} 404$ $\left(\mathrm{M}^{+}+\mathrm{H}\right), \quad 388, \quad 371, \quad 356, \quad 326, \quad 214,189,171,71$; HRMS (EI) calcd for $\mathrm{C}_{20} \mathrm{H}_{28} \mathrm{~F}_{3} \mathrm{NO}_{4}\left(\mathrm{M}^{+}+\mathrm{H}\right)$ 404.2048, found 404.2054.

Conversion of cis-11 into (R)-MTPA Ester cis-14 (cis-(R)-14): IR (neat) $v$ 3314, 2956, 2926, 2853, 1693, $1106 \mathrm{~cm}^{-1} ;{ }^{1} \mathrm{H}$ NMR $\left(400 \mathrm{MHz}, \mathrm{CDCl}_{3}\right) \delta 0.79(\mathrm{~d}, J=6.8$ $\mathrm{Hz}, 3 \mathrm{H}), 1.23(\mathrm{~d}, J=13.6 \mathrm{~Hz}, 1 \mathrm{H}), 1.48(\mathrm{dd}, J=7.2,13.2, \mathrm{~Hz}, 1 \mathrm{H}), 1.58(\mathrm{~d}, J=$ $14.4 \mathrm{~Hz}, 1 \mathrm{H}), 1.83(\mathrm{dd}, J=6.0,14.0 \mathrm{~Hz}, 1 \mathrm{H}), 2.01-2.09(\mathrm{~m}, 1 \mathrm{H}), 3.12(\mathrm{dd}, J=8.8$, $14.0 \mathrm{~Hz}, 2 \mathrm{H}), 3.31(\mathrm{~d}, J=8.8 \mathrm{~Hz}, 1 \mathrm{H}), 3.31(\mathrm{~d}, J=8.8 \mathrm{~Hz}, 1 \mathrm{H}), 3.30(\mathrm{~s}, 3 \mathrm{H}), 3.39$ 
(s, $1 \mathrm{H}), 3.49$ (bd, $J=1.6 \mathrm{~Hz}, 3 \mathrm{H}), 4.26-4.32(\mathrm{~m}, 1 \mathrm{H}), 7.36-7.39(\mathrm{~m}, 3 \mathrm{H})$, 7.64-7.66 (m, $2 \mathrm{H}), 8.00(\mathrm{bd}, J=8.8 \mathrm{~Hz}, 1 \mathrm{H}) ;{ }^{13} \mathrm{C} \mathrm{NMR}\left(100 \mathrm{MHz}, \mathrm{CDCl}_{3}\right) \delta$ 14.3, 37.1, 39.6, $39.9,46.5,54.1,55.1,58.9,59.3,77.8,79.3,122.4,125.3,127.5,128.1,129.0,133.5$, 165.2; LRMS (EI) $m / z 404\left(\mathrm{M}^{+}+\mathrm{H}\right), 388,371,356,326,214,189,171,71$; HRMS (EI) calcd for $\mathrm{C}_{20} \mathrm{H}_{28} \mathrm{~F}_{3} \mathrm{NO}_{4}\left(\mathrm{M}^{+}\right)$403.1970, found 403.1984.

Conversion of trans-11 into (S)-MTPA Ester trans-14 (trans-(S)-14): IR (neat) v 3315, 2956, 2925, 1684, $1105 \mathrm{~cm}^{-1} ;{ }^{1} \mathrm{H}$ NMR (400 MHz, $\left.\mathrm{CDCl}_{3}\right) \delta 1.02(\mathrm{~d}, J=6.4$ $\mathrm{Hz}, 3 \mathrm{H}), 1.16(\mathrm{dd}, J=8.2,12.9 \mathrm{~Hz}, 1 \mathrm{H}), 1.31(\mathrm{dd}, J=7.9,13.6 \mathrm{~Hz}, 1 \mathrm{H}), 1.85(\mathrm{dd}, J$ $=7.5,12.6 \mathrm{~Hz}, 1 \mathrm{H}), 1.85-1.95(\mathrm{~m}, 1 \mathrm{H}), 2.01(\mathrm{dd}, J=7.5,13.5 \mathrm{~Hz}, 1 \mathrm{H}), 3.18(\mathrm{dd}, J$ $=8.8,10.4 \mathrm{~Hz}, 2 \mathrm{H}), 3.21(\mathrm{~d}, J=8.7 \mathrm{~Hz}, 1 \mathrm{H}), 3.25(\mathrm{~d}, J=8.7 \mathrm{~Hz}, 1 \mathrm{H}), 3.32(\mathrm{~d}, J=$ $1.1 \mathrm{~Hz}, 1 \mathrm{H}), 3.43(\mathrm{bd}, J=1.5 \mathrm{~Hz}, 1 \mathrm{H}), 3.94(\mathrm{td}, J=7.5,16.2 \mathrm{~Hz}, 1 \mathrm{H}), 7.11(\mathrm{~d}, J=$ 8.5 Hz, $1 \mathrm{H})$, 7.37-7.39 (m, $3 \mathrm{H}), 7.52-7.54(\mathrm{~m}, 2 \mathrm{H}) ;{ }^{13} \mathrm{C}$ NMR (100 MHz, $\left.\mathrm{CDCl}_{3}\right) \delta 18.1,38.4,38.7,41.0,45.2,54.9,56.4,59.1,59.3,78.1,78.7,115.6,121.7$, 127.6, 128.3, 129.2, 132.9, 165.6; LRMS (EI) $m / z 404\left(\mathrm{M}^{+}+\mathrm{H}\right), 388,371,356,326$, 214, 189, 170, 71; HRMS (EI) calcd for $\mathrm{C}_{20} \mathrm{H}_{28} \mathrm{~F}_{3} \mathrm{NO}_{4}\left(\mathrm{M}^{+}\right)$403.1970, found 403.1967.

Conversion of trans-11 into (R)-MTPA Ester trans-14 (trans-(R)-14): IR(neat) $v$ 2926, 2873, 1656, 1519, 1163, $1107 \mathrm{~cm}^{-1} ;{ }^{1} \mathrm{H}$ NMR (400 MHz, $\left.\mathrm{CDCl}_{3}\right) \delta 0.95(\mathrm{~d}, J=$ $6.6 \mathrm{~Hz}, 3 \mathrm{H}), 1.13(\mathrm{dd}, J=8.4,13.1 \mathrm{~Hz}, 1 \mathrm{H}), 1.40(\mathrm{dd}, J=7.9,13.7 \mathrm{~Hz}, 1 \mathrm{H}), 1.76$ $(\mathrm{dd}, J=7.6,12.9 \mathrm{~Hz}, 1 \mathrm{H}), 1.80-1.87(\mathrm{~m}, 1 \mathrm{H}), 2.04(\mathrm{dd}, J=7.3,13.5 \mathrm{~Hz}, 1 \mathrm{H}), 3.18$ $(\mathrm{dd}, J=8.8,11.9 \mathrm{~Hz}, 2 \mathrm{H}), 3.19(\mathrm{~d}, J=8.7 \mathrm{~Hz}, 1 \mathrm{H}), 3.27(\mathrm{~d}, J=8.7 \mathrm{~Hz}, 1 \mathrm{H}), 3.32$ 
$(\mathrm{d}, J=2.8 \mathrm{~Hz}, 1 \mathrm{H}), 3.44(\mathrm{bd}, J=1.3 \mathrm{~Hz}, 1 \mathrm{H}), 3.95(\mathrm{td}, J=7.7,16.4 \mathrm{~Hz}, 1 \mathrm{H}), 7.05$ $(\mathrm{d}, J=8.7 \mathrm{~Hz}, 1 \mathrm{H}), 7.37-7.39(\mathrm{~m}, 3 \mathrm{H}), 7.55-7.57(\mathrm{~m}, 2 \mathrm{H}) ;{ }^{13} \mathrm{C} \mathrm{NMR}(100 \mathrm{MHz}$, $\left.\mathrm{CDCl}_{3}\right) \delta 18.2,38.5,38.7,40.9,45.2,54.9,56.3,59.1,59.3,78.1,78.8,122.3,125.2$, 127.5, 128.3, 129.2, 133.1, 165.6; LRMS (EI) $\mathrm{m} / z 404\left(\mathrm{M}^{+}+\mathrm{H}\right), 388,371,356,326$, 214, 189, 171, 71; HRMS (EI) calcd for $\mathrm{C}_{20} \mathrm{H}_{28} \mathrm{~F}_{3} \mathrm{NO}_{4}\left(\mathrm{M}^{+}\right)$403.1970, found 403.1974 . 